\title{
COHERENCE OF POLYNOMIAL RINGS
}

\author{
B. V. GREENBERG ${ }^{1}$ AND W. V. VASCONCELOS ${ }^{2}$
}

\begin{abstract}
The main result is that $A[X]$, the polynomial ring in any number of indeterminates over a coherent ring $A$ of global dimension two, is coherent.
\end{abstract}

1. Introduction. All rings considered here are commutative and have an identity element. All modules are assumed unital and ring maps take the identity to the identity. A local ring is a ring with a unique maximal ideal.

A ring is said to be coherent if all of its finitely generated ideals are finitely presented. In too many ways these rings differ from noetherian rings, most strikingly in the failure of the "basis theorem": If a ring $A$ is coherent then $A[x]$-the polynomial ring in one indeterminate over $A$-is not necessarily coherent [11]. Here we show, however, that if the deep structure of the coherent ring is "sufficiently" rich, then the coherence of the polynomial ring over $A$ ensues. Precisely, it is shown that if $A$ is a coherent ring of (1) weak global dimension one of (2) global dimension two, then $A\left[x_{1}, \ldots, x_{n}\right]$ is coherent. The proof follows a track opened up by a sharp remark of Gruson on the use of generic flatness and uses the structure theory of rings of global dimension two as developed in [3], [10], [12]. A feature of the proofs is that they do not proceed by induction on the number of indeterminates, but in a manner to lend credence to the following conjecture: If $A[x]$ is coherent, then $A\left[x_{1}, \ldots, x_{n}\right]$ is also coherent.

2. Gruson's key remark. Let $A$ be a commutative ring, $B$ an $A$-algebra of finite presentation and $E$ a finitely generated $B$-module. As in [5, (3.4.6)] we attempt to determine the nature of $U$, the set of points in $\operatorname{Spec}(B)$ where $E$ is $A$-flat, and whether $E$ admits a finite $B$-presentation over $U$. The following modification of [loc. cit.] was given by Gruson.

(2.1) Proposition. Let $A, B$ and $E$ be as above and let $h: A \rightarrow C$ be an injective ring homomorphism. Assume $E_{C}=E \otimes_{A} C$ is of finite presentation over $B_{C}=B \otimes_{A} C$. Then the set $U$ of points of $\operatorname{Spec}(B)$ where $E$ is $A$-flat is open and $E$ is of finite presentation over $U$.

Proof. Given a point $P$ of $U$ let us find a neighborhood of $P$ where the

Received by the editors February 20, 1975.

AMS (MOS) subject classifications (1970). Primary 13B25; Secondary 13C10, 13D05.

Key words and phrases. Projective dimension, global dimension, Tor-dim, coherent ring, polynomial ring.

${ }^{1}$ The first author thanks the University of Nebraska for their support and fine hospitality while visiting there during 1973-1974.

${ }^{2}$ The second author was partially supported by National Science Foundation Grant GP33133 . 
assertion is valid. By restricting $\operatorname{Spec}(B)$ as in [loc. cit.], we can find a surjection of $B$-modules, $f: F \rightarrow E$, where $F$ is of finite presentation and $A$-flat and $f_{P}$ is an isomorphism. Since $F_{C}$ is of finite presentation we may assume, restricting $\operatorname{Spec}(B)$ still further if necessary, that $f \otimes_{A} C$ is an isomorphism. We deduce the assertion from the commutative diagram

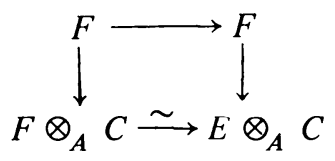

where the left vertical map is injective by the flatness of $F$.

3. Some consequences for stable (= polynomial) coherence. In all the cases we consider, $A$ is a ring whose total ring of quotients, $K$, is von Neumann regular.

(a) $A$ is von Neumann regular. Let $I$ be a finitely generated ideal of $B=A\left[x_{1}, \ldots, x_{n}\right]$. Then $E=B / I$ is a $B$-module of finite presentation and $E$ and $B$ are $A$-flat. It follows from the principle of infinite finite presentation $[4,(11.3 .9 .1)]$ that $I$ is finitely presented. This result has also been proved in [9].

(b) $A$ is semihereditary. If $B=A\left[x_{1}, \ldots, x_{n}\right]$ and $I$ is a finitely generated ideal of $B, I$ is $A$-flat. Taking $C=K$ in Proposition (2.1) we get that $I$ is finitely presented.

Thus far we have shown that coherent rings of weak global dimension (= Tor-dim for euphony) less than or equal to one are stably coherent. The next order of complexity will be considered in the next section.

4. Rings of global dimension two. Let $A$ be a ring of global dimension two having total ring of quotients $K$. Then $A$ is coherent if and only if $K$ is von Neumann regular [13]. We will show that $A\left[x_{1}, \ldots, x_{n}\right]$ is coherent by first reducing to the local case and then giving a proof in that situation.

(i) Reduction to the local case. Let $B$ and $I$ be as above. If $P$ is a prime ideal of $B$ where $I$ is not $A$-flat, then $p=A \cap P$ is such that $A_{p}$ is not a valuation ring, and so $p$ is a maximal ideal of $A$ [12] and is, according to [10], finitely generated. Thus $V$, the set of prime ideals in $B$ where $I$ is not $A$-flat, is closed in $\operatorname{Spec}(B)$ by $(2.1)$ and the primes in $V$ are finitely generated. As noted in [14], $V$ is then a Noetherian space and is then the union of finitely many irreducible components, $V=V\left(P_{1}\right) \cup \cdots \cup V\left(P_{n}\right)$. Let $p_{i}=P_{i} \cap A$ and let $J=p_{1} \cdots p_{n} \subset A$. Now $J$ is finitely generated, say $J=\left(f_{1}, \ldots, f_{m}\right)$ (actually $J$ can be generated by three elements [10]), and consider the change of rings determined by the standard map

$$
A \rightarrow A_{p_{1}} \times \cdots \times A_{p_{n}} \times A_{f_{1}} \times \cdots \times A_{f_{m}} .
$$

This map is faithfully flat and in a component such as $A_{f_{i}}, I_{f_{i}}$ is $A$-flat (and of finite presentation).

To complete the proof of the coherence of $B$, it suffices to see that $I \otimes_{A} A_{p_{i}}\left[x_{1}, \ldots, x_{n}\right]$ is finitely presented over $A_{p_{i}}\left[x_{1}, \ldots, x_{n}\right]$ [2, Chapter I, $\S 3$, Proposition 11]. Thus we need only to prove that if $A$ is a local ring of global dimension two (necessarily coherent [12]), then $A\left[x_{1}, \ldots, x_{n}\right]$ is coherent. 
(ii) The local case. Before focusing our attention on the coherence of the polynomial ring over a local ring of global dimension two, we will prove a proposition concerning coherence in Cartesian squares. Then drawing upon the structure theory of local rings of global dimension two, we will apply this result to the question at hand.

A commutative square of ring homomorphisms

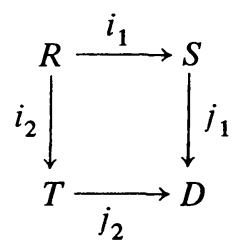

is said to be cartesian (or a pull-back) if $R$ is the product of $A$ and $T$ over $D$. Letting $j_{1}$ and $i_{2}$ be onto and $i_{1}$ and $j_{2}$ be one-one, we can identify $H=\operatorname{ker}\left(i_{2}\right)$ with an ideal in $S$, that is, $H=H S$. Assume further that $H$ is a flat ideal of $R$ and $S$ is flat as an $R$-module.

(4.1) Proposition. Adopting the format above, if $S$ is coherent and $T$ is noetherian, then $R$ is coherent.

Proof. Consider the usual ring homomorphism $R \rightarrow T \times S$ and note that the image of $H$ is $(0, H)$, so $H$ is an ideal common to $R$ and $T \times S$.

Let $L=\left(\alpha_{1}, \ldots, \alpha_{n}\right)$ be an ideal in $R$ and consider the exact sequence

$$
0 \rightarrow K \rightarrow R^{n} \stackrel{f}{\rightarrow} L \rightarrow 0
$$

where $f$ takes the $i$ th unit vector to $\alpha_{i}$ and $K=\operatorname{ker}(f)$.

Tensoring (*) with $S$, we get that $K \otimes S$ is finitely generated as an $S$-module since $L \otimes S$ is a finitely generated ideal of $S$ and $S$ is coherent and flat as an $R$-module. Tensoring (*) with $T$, we get that

$$
0 \rightarrow K \otimes T \rightarrow T^{n} \stackrel{f \otimes 1}{\rightarrow} L \otimes T \rightarrow 0
$$

is also exact since $\operatorname{Tor}_{1}^{R}(R / H, L)=0$ because $H$ is a flat ideal and $L$ is a submodule of a free $R$-module.

Since $T$ is noetherian, $K \otimes T$ is a finitely generated $T$-module so $K \otimes$ $(T \times S)$ is a finitely generated $(T \times S)$-module. However, [15, (1.2)] provides a descent of finite generation criteria applicable. Thus, $K$ is finitely generated, so $R$ is coherent.

As shown in [3, (3.4)], if $A$ is a local ring of global dimension two, then $A$ can be embedded in a cartesian square

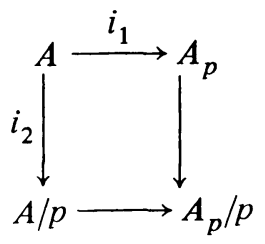

where $p$ is a flat prime ideal of $A, A_{p}$ is a valuation domain and $A / p$ is noetherian. 
(4.2) Theorem. If $A$ is a local ring of global dimension two, then $A\left[s_{1}, \ldots, x_{n}\right](=A[X])$ is coherent.

Proof. If we tensor the cartesian square above with $A[X]$ we get the cartesian square

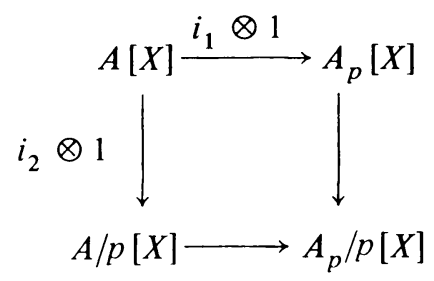

where $\operatorname{ker}\left(i_{2} \otimes 1\right)=p[X]$ and $p[X]$ is a flat prime ideal in $A[X]$.

According to $[5,(3.4 .7)], A_{p}[X]$ is coherent, and since $A / p[X]$ is noetherian, $A[X]$ is coherent by the proposition.

Combining the analysis in part (i) above with Theorem (4.2), we get

(4.3) TheOREM. If $A$ is a coherent ring of global dimension two, then $A\left[x_{1}, \ldots, x_{n}\right]$ is coherent.

Drawing upon [2, Chapter I, §2, Example 12] we have

(4.4) Corollary. If $A$ is a coherent ring of global dimension two, then the polynomial ring in any number of indeterminates over $A$ is also coherent.

5. Similarities and difficulties in Tor-dim two. Here we consider some aspects of the theory of coherent local rings of Tor-dim two and contrast them to similiar facets of rings of global dimension two. It will serve the purpose of pointing out the difficulties in attempting to prove coherence in polynomial extensions in general. In order to have a proof similiar to that of $\S 4$, a lot more about the structure of rings of Tor-dim two than is currently known would have to be available.

(5.1) In global dimension two, local rings are automatically coherent. This is no longer the case in Tor-dim two as the following construction showsused by several authors, and particularly by B. Osofsky to provide examples of local rings of finite global dimensions but with zero-divisors.

Let $V$ be a valuation domain with nonprincipal maximal ideal $M$. Consider the cartesian square

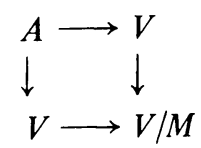

where the maps onto $V / M$ are the natural ones. Then $A$ is the subring of $B=V \times V$ consisting of pairs $(a, b)$ such that $a-b \in M$. Note that $A$ is a local ring having zero-divisors and maximal ideal $N=\Lambda ! \times M, N$ is the conductor of $B$ in $A$ and $A / N \simeq V / M$.

(5.2) TheOREM. Adopt the situation above and assume further that $\mathrm{gl} \operatorname{dim} V$ $=n<\infty$. Then

$$
\text { gl } \operatorname{dim} V \leqslant \mathrm{gl} \operatorname{dim} A \leqslant \mathrm{gl} \operatorname{dim} V+1 .
$$


Proof. Assume we have shown that $\mathrm{wd}_{A} B \leqslant 1$, and let

$$
0 \rightarrow L \rightarrow P_{n-1} \rightarrow \cdots \rightarrow P_{0} \rightarrow I \rightarrow 0
$$

be a resolution for the ideal $I$ of $A$ where $P_{i}$ is $A$-projective. To see that $L$ is also projective, tensor ( $*$ ) with $B$ to get that $(*) \otimes B$ yields a $B$-projective resolution of $I \otimes B$ and so [15, (1.1)] applies since $N$ is the conductor of $B$ in $A$ and $L \otimes A / N$ is $A / N$ free.

To see that $\operatorname{wd}_{A} B \leqslant 1$, it is enough to show $\operatorname{Tor}_{1}^{A}(I, B)=0$ for every finitely generated ideal $I$ of $A$, say $I=\left(\left(a_{1}, b_{1}\right), \ldots,\left(a_{m}, b_{m}\right)\right)$. If $v$ denotes the valuation associated to $V$, by consideration of $\min \left\{v\left(a_{1}\right)\right\}$ one can conclude that $I=(a, 0) A \oplus(0, b) A$ for appropriate $a, b \in V$. We can then assume that $I=(a, 0) A$ and so a resolution of $I$ is

(* *) $\quad 0 \rightarrow(0, M) A \rightarrow A \rightarrow(a, 0) A \rightarrow 0$.

Tensoring $(* *)$ with $B$ we get

$$
0 \rightarrow \operatorname{Tor}_{1}^{A}(I, B) \rightarrow(0, M) \otimes B \rightarrow B \rightarrow I \otimes B \rightarrow 0 .
$$

Letting $x \in \operatorname{Tor}_{1}^{A}(I, B)$ we may assume $x=(0, d) \otimes(c, 0)$. Because $M$ is not principal, we can write $(0, d)=(0, f) \cdot(0, e)$ for $f, e \in M$. Thus

$$
x=(0, f)(0, e) \otimes(c, 0)=(0, f) \otimes(0, e)(c, 0)=0 ;
$$

hence, $\operatorname{Tor}_{1}^{A}(I, B)=0$.

(5.3) REMARKS. (i) If $V$ is a valuation domain with countably generated ideals (i.e., gl $\operatorname{dim} V=2$ [7]) with a nonprincipal maximal ideal $M$, then we see $A$ has global dimensions less than or equal to three. But it cannot be two since $A$ is not a domain, so $\mathrm{gl} \operatorname{dim} A=3$.

(ii) Since $(0, M)$ is a flat ideal of $A$, by examining $(* *)$ we see that every finitely generated ideal of $A$ has flat dimension less than or equal to one. Since $A$ is not a valuation domain, Tor-dim $A=2$.

(iii) Since local coherent rings of finite Tor-dim are domains [1], [16], the ring $A$ is not coherent. Incidentally, there exist domains of Tor-dim two that are not coherent.

(5.4) In trying to discover some structural features of a local coherent ring of Tor-dim two, $(A, M)$, we might, as in [12], examine the vector space $M / M^{2}$ over $A / M$.

(5.5) TheOREM. If $k$ is the dimension of $M / M^{2}$ over $A / M$, then $k=0,1$ or 2. If $k=0$ or 1 then $M$ is not finitely generated. If $k=2$ then $M$ can be generated by two elements.

Proof. Observe first as in [8], [12] that $A$ is a GCD-domain since every doubly generated ideal, $(a, b)$, has a resolution of the form $0 \rightarrow A \rightarrow A^{2} \rightarrow(a$, $b) \rightarrow 0$. If $M$ is finitely generated then $k \neq 0$ or 1 since $A$ is neither a field nor a valuation domain.

Suppose $x_{1}, x_{2}, x_{3}$ are three elements of $M$, with at least one of them not in $M^{2}$. If they are linearly independent modulo $M$, a minimal resolution of the ideal $I$ they generate is of the form $0 \rightarrow A^{2} \stackrel{\phi}{\rightarrow} A^{3} \rightarrow I \rightarrow 0$. By Burch's theorem [6, p. 148], $I$ would be generated by the $2 \times 2$ minors of the matrix for $\phi$ and would lie in $M^{2}$. 
(5.6) Remark. Note that for any $y \in M \backslash M^{2}, A /(y)$ is a valuation domain since $(y)$ is a prime ideal, $y$ being indecomposable in a GCD-domain and Tor-dim $A /(y)=1$ [8].

(5.7) ExAmples. (i) Let $V$ be a valuation domain with maximal ideal $N$ and let $A=V[x]_{(x, N)}$. Note that $A$ is coherent [5, (3.4.7)], and Tor-dim $A \leqslant 2$. Since the maximal ideal of $A, M=(x, N)$, is not flat, $A$ is not a valuation domain so Tor-dim $A=2$. We have that $k=2$ or 1 according to whether $N$ is principal or not.

(ii) (a case where $k=0$ ) Let $F$ be a field and consider the sequence of polynomial rings $F[x, y] \subset F\left[x^{1 / 2}, y^{1 / 2}\right] \subset F\left[x^{1 / 4}, y^{1 / 4}\right] \subset \cdots$ and let $S$ be the union. Note that each of the rings is free as a module over the preceding so $S$ is coherent and Tor-dim $S \leqslant 2$. Let $A$ be the localization of $S$ at the origin, so Tor-dim $A \leqslant 2$ and $A$ has maximal ideal $M=M^{2}$. The elements $x$ and $y$ are however without a common divisor other than a unit. Thus $A$ is not a valuation domain so $\operatorname{Tor}-\operatorname{dim} A=2$.

(5.8) Remark. A key fact used in $\$ 4$ was that in a ring $A$ of global dimension two, for a nonmaximal prime ideal, $A_{p}$ is a valuation domain. This is no longer the case in rings of Tor-dim two as the first example above attests. This seems to be a main reason for the failure of attempts to show the coherence of $A[x]$ for $A$ coherent of Tor-dim two.

\section{REFERENCES}

1. J. Bertin, Anneaux cohérents réguliers, C. R. Acad. Sci. Paris Sér. A-B 273(1971), A1-A2. MR43 \#4820.

2. N. Bourbaki, Éléments de mathématique. Fasc. XXVII. Algèbre commutative. Chap. 1: Modules plats. Chap. 2: Localisation, Actualités Sci. Indust., no. 1290, Hermann, Paris, 1961. MR36 \#146.

3. B. Greenberg, Global dimension of cartesian squares, J. Algebra 32(1974), 31-43.

4. A. Grothendieck and J. Dieudonné, Éléments de géométrie algébrique. IV. Étude locale des schémas et de morphismes de schémas. III, Inst. Hautes Études Sci. Publ. Math. No. 28(1964). MR36 \#178.

5. L. Gruson and M. Raynaud, Critères de platitude et de projectivité. Techniques de "platification" d'un module, Invent. Math. 13(1971), 1-89. MR46 \#7219.

6. I. Kaplansky, Commutative rings, Allyn and Bacon, Boston, Mass., 1970. MR40 \#7234.

7. B. L. Osofsky, Global dimension of valuation rings, Trans. Amer. Math. Soc. 127(1967), 136-149. MR34 \#5899.

8. Y. Quentel, Sur le théorème d'Auslander-Buchsbaum, Colloque d'Algèbre Commutative, Univ. de Rennes, 1972.

9. G. Sabbagh, Coherence of polynomial rings and bounds in polynomial ideals, J. Algebra 31(1974), 499-507.

10. H. Sheng, Finiteness of prime ideals in rings of global dimension two, Proc. Amer. Math. Soc. 41(1973), 363-369. MR48 \#6081.

11. J.-P. Soublin, Anneaux et modules cohérents, J. Algebra 15(1970), 455-472. MR41 \#5422.

12. W. V. Vasconcelos, The local rings of global dimension two, Proc. Amer. Math. Soc. 35(1972), 381-386. MR46 \#7230.

13. , Rings of global dimension two, Conf. on Commutative Algebra (Kansas, 1972), Springer-Verlag, Berlin and New York, 1973, pp. 243-251.

14. Coherence of one polynomial ring, Proc. Amer. Math. Soc. 41(1973), 449-456.

MR48 \#3955.

15. , Conductor, projectivity and injectivity, Pacific J. Math. 48(1973), 603-608. MR48 \#284.

16. __ Finiteness in projective ideals, J. Algebra 25(1973), 269-278. MR47 \#3378.

Department of Mathematics, University of Virginia, Charlottesville, Virginia 22903

Department of Mathematics, Rutgers University, New Brunswick, New Jersey 08903 